\title{
- Insights into Biomedical 3D Printing
}

\section{Prachi Khamkar}

IJCRR

Section: Healthcare ISI Impact Factor (2019-20): 1.628

IC Value (2019): 90.81

$\operatorname{SJIF}(2020)=7.893$

Department of Pharmaceutics, University of Mumbai, Mumbai, Maharashtra, India.

3D printing technology appraised as one of the emerging field in Pharmaceuticals and Healthcare sector. Individualization of the therapeutic method enables 3D modelling to pave the way for the diagnosis of uncommon diseases. Although the mass processing of tablets is unnecessary, it would be possible to minimize financial expenses and time for performing medication clinical trials by manufacturing formulations in small amounts. It is now possible to produce personalised dosage form and replicas of prosthetics and tailored made devices. Therefore, transformative 3D printing technology can open new window of opportunities that were previously underestimated. Pharmaceutical scientist has made rapid advances in 3D printing, which can innovate the medicine because of its strong versatility and potential. $3 \mathrm{D}$ helps to fabricate a variety of complex dosage form with different dimensions and polymers. The development of 3D Printing and its use in the pharmaceutical industry also resulted in a fundamental change in terms of personalisation. The innovations and advances across the last decades point to a promising future for improving patient-specific medication delivery technologies, spanning from oral treatment formulations to environmentally friendly scaffolds.

The most widely used 3D printing technique is Material extrusion method, which is the most accessible and least expensive type of 3D printing technology worldwide. They are also referred to as Fused Deposition Modelling or FDM. Additionally, they are often referred to as Fused Filament Fabrication or FFF. It operates by loading a spindle of filament into the 3D printer and feeding it via a printer nozzle located in the extrusion head. The printer's heater is turned on, and the motor drives the filament into the hot nozzle. The printer then pushes the extrusion head in the specified direction, depositing the molten content on the constructed layer, where it cools and solidifies. When one layer is printed on base, the printer continues to print the next layer. This method of printing cross-sections is replicated until the shape is fully shaped. The printer then pushes the extrusion head in the specified direction, depositing the molten content on the constructed layer, where it cools and solidifies. If a layer is full, the printer moves on to the next layer until the object is completely formed.

Let's concentrate on 3DBio-printing, the object is generated using a computer-aided design (CAD) software system and then exported as a print-ready format. The exported file divides the three-dimensional object into layers that are then printed layer by layer. The technology entails the layer-bylayer printing of a mixture of materials, controlling each layer for its form, ultimately creating a dynamic 3D system with minimal spatial arrangement constraints. Bio-printing technology has recently evolved and includes printing traditional biomaterials and live cells onto intricate tissue and organ structures (typically referred to as "bioink"). Numerous Bioprinting techniques for depositing cells and Hydrogels simultaneously have been established in recent years, which include printing of heart valves, liver cells etc. Initially, cell printers were adapted from widely available desktop inkjet printers that were used to print polymers. In these printers, cell suspensions or aggregates are inserted in a printer extruder device, and a machine precisely controls the printing process. The valve-based droplet ejection mechanism is another significant updated technique for developing cellencapsulating hydrogel beads. The valve opening time and actuation frequency are changed to regulate the number of droplets, scale, and number of cells in a single droplet.

One of the attention-grabbing terms in recent years is 3D printed Skin, the beauty and skincare industries; there is a massive market for synthetic skin. These players such as L'Oréal and P\&G depend on bioprinted 3D skin for product testing. Companies in the cosmetics/pharmaceutical industry will have to conduct tests on in vitro before going ahead of animal models, which were formerly the primary options, but now they can experiment on 3D printed skin as mimics the human skin. Bioprinted skins could be used to heal burned injuries and wounds; cells from the patient's body can be employed in the bioprinting of the skin. 
Let us focus on Regulation in the 3D printing industry, Even though it has enormous potential and plays a major role in the modern economy, however, there is no coherent global strategy regarding 3D Printing. In the developed countries, the law seems to be targeted to particular areas such as medical equipment, although in countries where it has not, it is wide more and more businesses and policymakers are increasingly looking at 3D printing as a tool for the future, although governments must rely on regulatory sectorspecific models where the situation calls for it. Nowadays, due to the advent of 3D printing in various sectors of the economy, the use of 3D printing differs considerably from country to country. While current guidance from the Centre for Drug Evaluation and Research (CDER) is lacking, these drugs are controlled by the same pathways as their generic counterparts. There are special compliance issues to consider for and product class of entity. Centre for Biologics Evaluation and Research (CBER) is doing its own studies to further understanding of 3D printing's function in drug development and has also been working with manufacturers to help with this new technology. Researchers have also spoken with the CBER constituents, who are exploring the usage of $3 \mathrm{D}$ printing of human tissues. A former FDA commissioner said in 2017 that it will begin reviewing the regulatory concerns around bio-printing to see whether more advice was needed outside of the regulations. To date, however, no additional changes have been released.

So, the medical world moves into personalized medicine, 3D printing will offer prescription dosage forms tailored to each patient. Moreover, a cost-efficient approach for the manufacture of bulk manufacturing dosage forms can be possible by $3 \mathrm{D}$ printing. The regulatory system applicable to $3 \mathrm{D}$ printed devices is identical to that applicable to conventionally manufactured devices, except that $3 \mathrm{D}$ printed devices are used almost immediately after manufacturing, exempting lengthy quality assurance procedures. Therefore, the production mechanism should be set up to allow for better quality management, thus eliminating quality assurance and monitoring afterwards.

Keywords: 3 D printing, 3D printed Skin, Material extrusion method, 3DBio-printing, Fused Deposition Modelling, Fused Filament Fabrication

\section{REFERENCE}

1. Al-Dulimi Z, Wallis M, Tan D, Maniruzzaman M, Nokhodchi A. 3D printing technology as innovative solutions for biomedical applications. Drug Discovery Today. 2021;26(2):360-383. 